
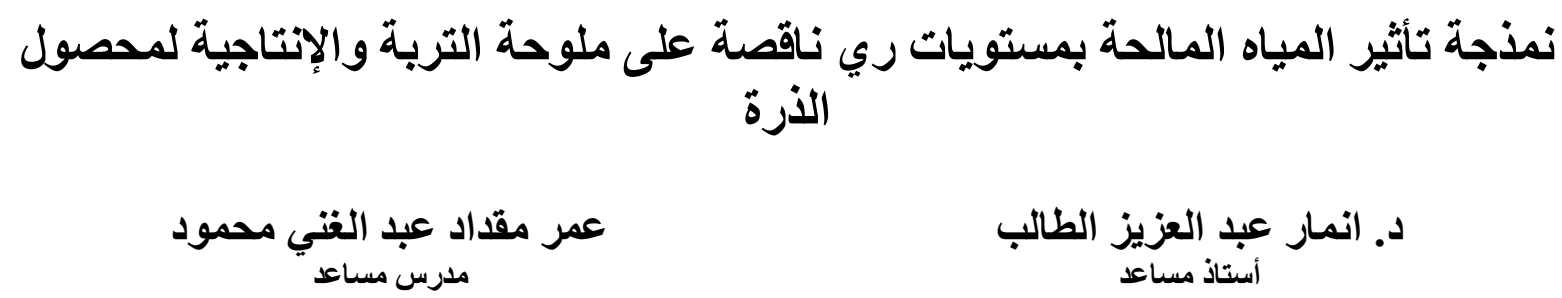

المستخلص

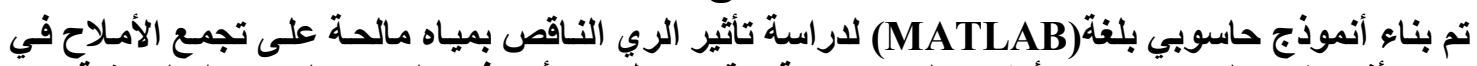

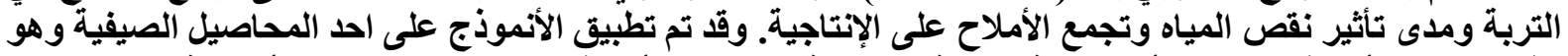

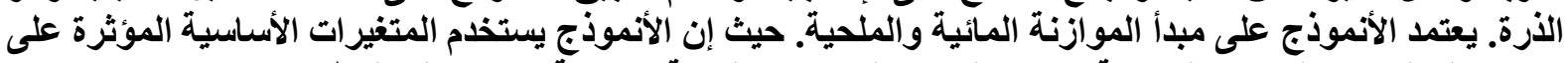

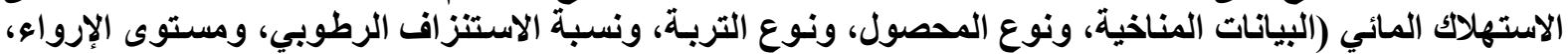

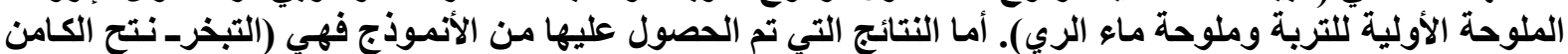

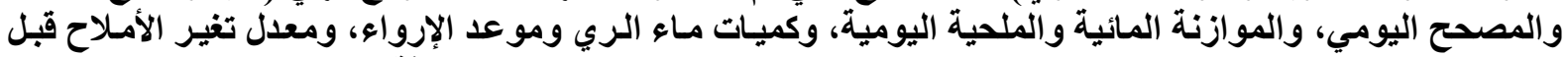

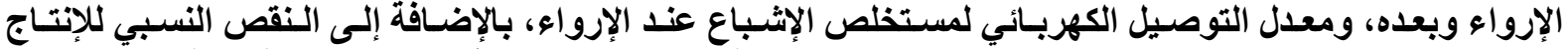

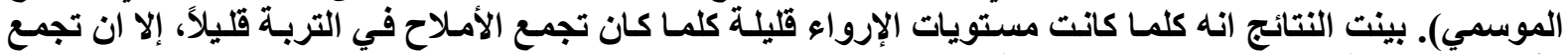

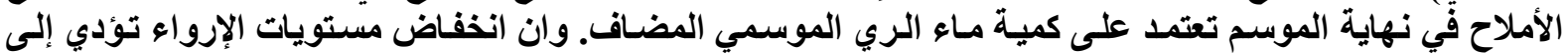

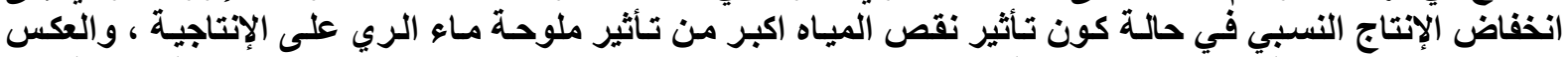

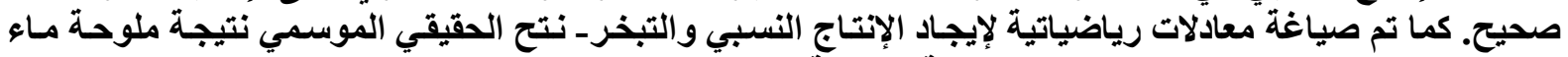

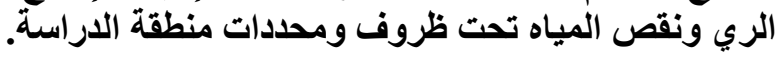

\title{
Modeling Effect of Saline Water With deficient Irrigation levels on Soil Salinity and Yield for Maize
}

\author{
Dr . A. A. A. AL-Talib \\ Ass. Professor \\ O. M. A. G. Mahmood \\ Ass. lecture
}

\section{Abstract}

Computer model is made in (MATLAB) language to study the effect of deficit irrigation with saline water on the salts accumulation in soil and the effect extent of the lack of water and the salts accumulation on yield. The Model has been applied on summer crop, Maize, and it relies on the principle of balancing water and salt. The Model are uses the basic variables entered in the irrigation (climate data, crop type, soil type, and the rate of moisture depletion, irrigation level, the initial soil salinity and salinity of irrigation water). The results obtained from the program are (daily potential and adjusted Evapotranspiration, daily balance of water and salt, the quantities of irrigation water and Perfusion appointment, The rate of change of concentration of salts before and after Perfusion, electric conductivity of saturation extract at Slaking, calculating the relative defect of seasonal yield). study show when using a little amount of irrigation water we have a little salt accumulation in the soil, but the salt accumulation at the end of the season depends on the gross depth of irrigation water added . Low levels of irrigation led to lower proportional yield in the event that the impact of water shortage was bigger than the impact of irrigation water salinity on productivity, and vice versa. Formulation of mathematical equations to find the relative yield and seasonal actual Evapotranspiration because of irrigation water salinity and water shortage, under the condition of study area and determinants.

Keywords: deficit, salinity, Yield, depletion, Evapotranspiration

$$
\text { قبل: } 2010 \text { - } 73 \text { - }
$$

أستلم: 2010 - 3 - 38 


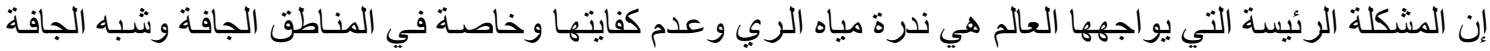

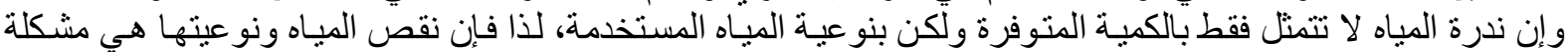

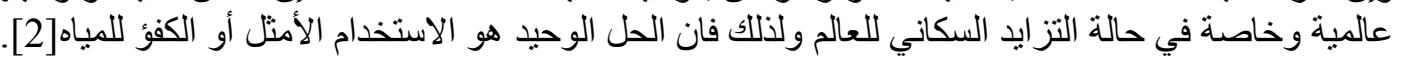

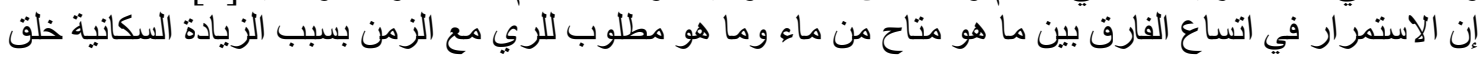

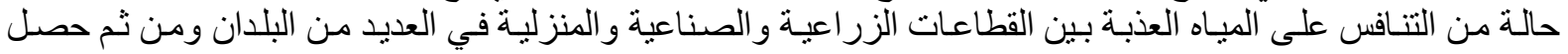

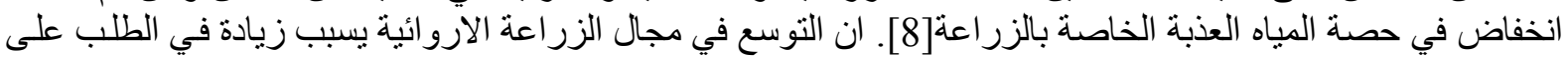

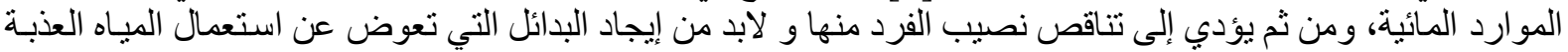
لسد جزء من العجز المائي المنوقع.

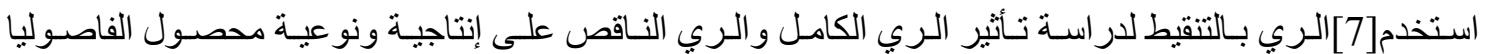

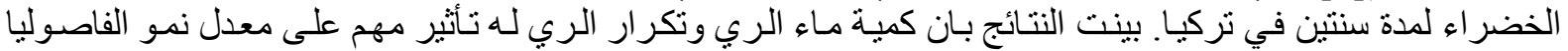
الخضر اء و إنتاجيتها تحت شروط مناخ البحر المتوسط في تركيا.

أجرى[5]تجارب حقلية في تكساس لتقيبم إنتاجية محصول القطن عند استخدام الماء المالح بمسنتوى نـاقص. وبينت

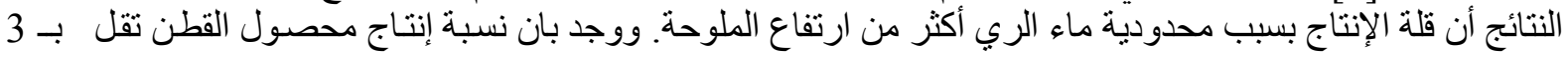

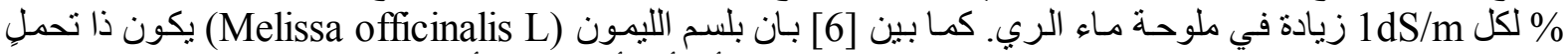
عالِ لجهد الماء و تحمل منوسط للملوحة من خلال تجارب حقلية، أي أن تأثير جهد الأملاح على إنتاجية بلسم الليمون اكبر ذلِ من جهد الماء.

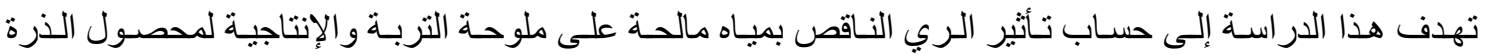

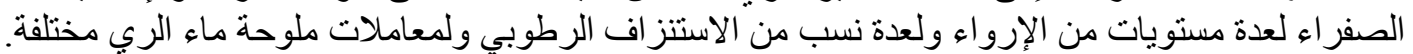

طريقة البحث

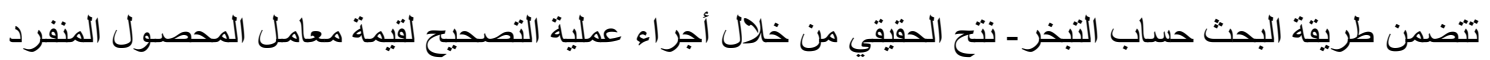

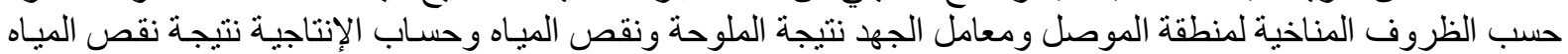
و ملوحة المياه.

\section{التبخر- نتح الحقيقي للمحصول ET}

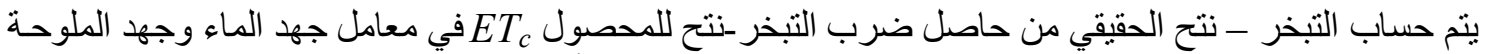

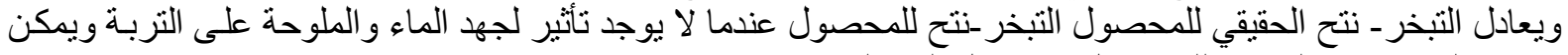

$$
E T_{C \text { adj }}=E T_{c} \times K_{W} \times K_{E c e}
$$
: ET

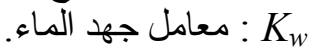

ئ日

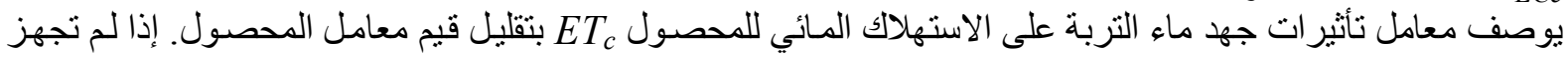

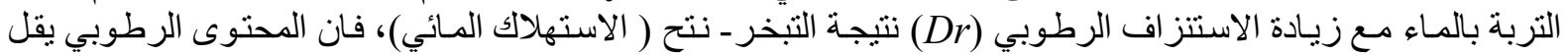

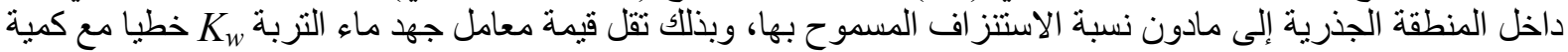

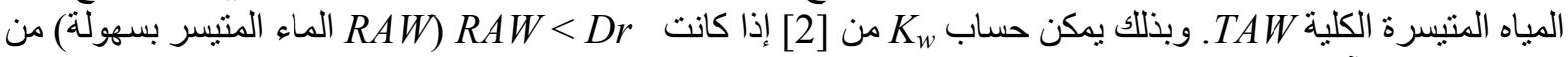

$$
K_{W}=\frac{(T A W-D r)}{(T A W-R A W)}=\frac{(T A W-D r)}{(1-P) T A W}
$$


TAW : الماء المتيسر الكلي في المنطقة الجذرية (ملم) : P : نسبة الاستنز اف الحرج بقيمة 0.52 عند حصول جهد للملوحة في التربة، يمكن استخدام المعادلة (3) لـ [2].

$K_{E c e}=1-\frac{b}{K_{Y} 100}\left(E C e-E C e_{\text {threshold }}\right)$

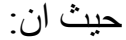

(KC

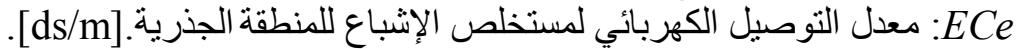
ECe threshold .[ds/m]. $Y_{m}$ b: النقص في الإنتاجية على وحدة الزيادة في ECe E ولحساب التبخر - نتح للمحصول نضرب معندأمل المحصول في التبخر - نتح المرجعي كما في معادلة(4) لـ[2].

$E T_{c}=E T_{o} K_{c}$

ET。

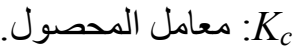

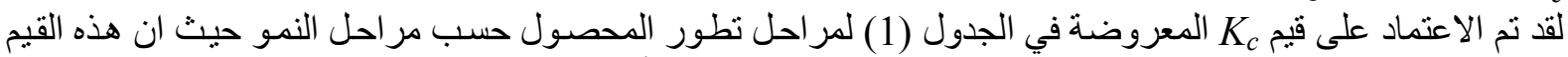

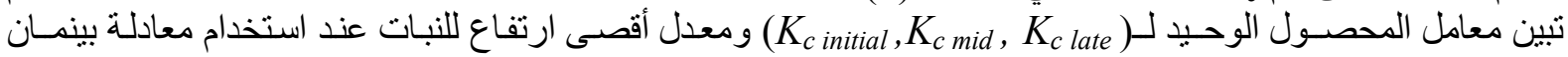
مونتيث عند الظروف المناخية القياسية (U)=2m/s , RHmin=45\%) .

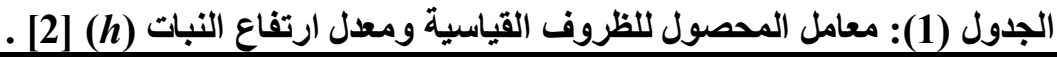

\begin{tabular}{|c|c|c|c|c|}
\hline h) & النهائي(المحلي & 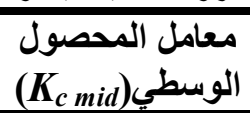 & الأولي(مامل المحصول) & المحصول \\
\hline 2 & 0.6 & 1.2 & 0.3 & الأرة \\
\hline
\end{tabular}

لذلك تحتاج هذه القيم المأخوذة من الجدول (1) إلى تصحيح وكالآتي:

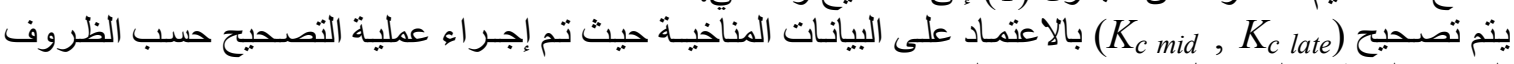

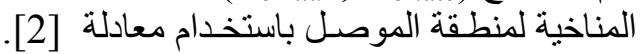

$K_{c(\text { mid }, \text { late })}=K_{c(\text { table })}+\left[0.04 \times\left(U_{2}-2\right)-0.004\left(R H_{\min }-45\right)\right] \times\left(\frac{h}{3}\right)^{0.3}$

K(mid,late)

(1Table)

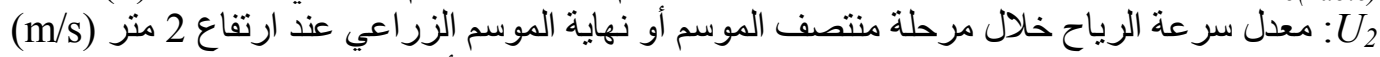

: RH $H_{\text {min }}$ h: معدل ارتفاع النبات (م) من [2].

الجدول (2): قيم معامل المحصول المصححة لمراحل النمو.

\begin{tabular}{|c|c|c|c|}
\hline $\begin{array}{c}\text { معامل المحصول } \\
\text { النهائي } \\
\left(\boldsymbol{K}_{\text {c late }}\right)\end{array}$ & معامل المحصول & 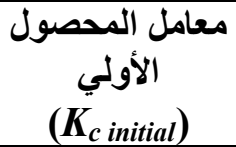 & المحصول \\
\hline 0.67 & 1.29 & 0.3 & الأزرة \\
\hline
\end{tabular}




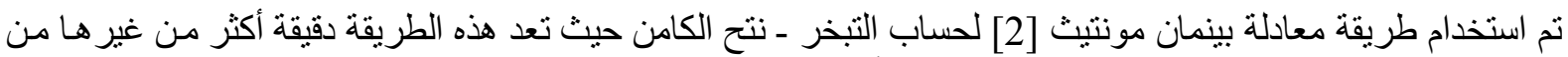
الطر ائق ويمكن تطبيقها على البيانات المناخية الكاملة أو الناقصة حيث تكون صيغة معادلة بينمان مونتيث كالآتي.

$$
E T_{\circ}=\frac{0.408 \Delta\left(R_{n}-G\right)+\gamma \frac{900}{T+273} U_{2}(e s-e a)}{\Delta+\gamma\left(1+0.34 U_{2}\right)}
$$

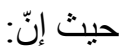

$$
\begin{aligned}
& \text { ET。 }
\end{aligned}
$$

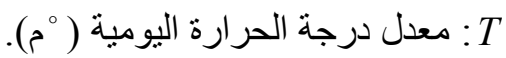

$$
\begin{aligned}
& \text { Rn } R_{n} \\
& \text { G : كثافة تدفق حر ارة التربة (ميكاجول/م }{ }^{2} \text { يوم). } \\
& \text { es : ضغط بخار التشبع (كيلو باسكال). } \\
& \text { ea : ضغط البخار الفعلي ( كيلو باسكال). }
\end{aligned}
$$

حساب الملوحة قبل الإرواء وبعده:

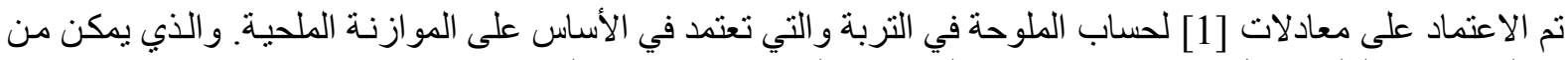

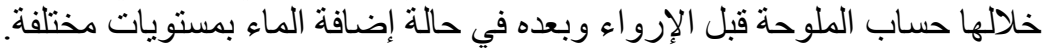

$E C s b i=E C s a i \times\left(\frac{T W A T-D_{\text {rinitial }}}{T W A T-D_{r}}\right)$

$E C s a i=\frac{E C s b i \times\left(T W A T-D_{r}\right)+\left(D_{r}-D_{r} \text { initial }\right) \times E C i}{\left(T W A T-D_{r} \text { initial }\right)}$

ECsbi TWAT

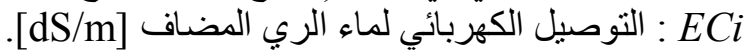
ECsai Drinitial

\section{النقص بالإنتاج}

توصلت منظمة الغذاء و الزر اعة الدولية [4] إلى طريقة لتقدير النقص بالإنتاج. وذلك عن طريق حسياب الموازنـة

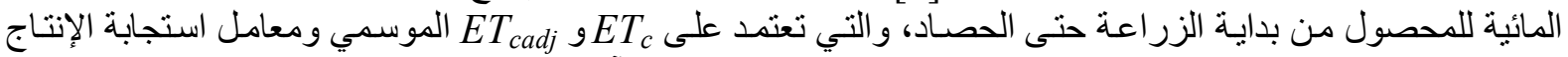
للماء. وقدمت هذه المنظمة معادلة لحساب نسبة النقص بالإنتاج بالصيغة الآنية: 


$$
\left(1-\frac{Y_{a}}{Y_{m}}\right)=K_{Y}\left(1-\frac{E T_{c a d j}}{E T_{c}}\right)
$$

حيث

$Y_{a}$

Y K

\section{تغير عمق المنطقة الجذرية خلال نمو المحصول}

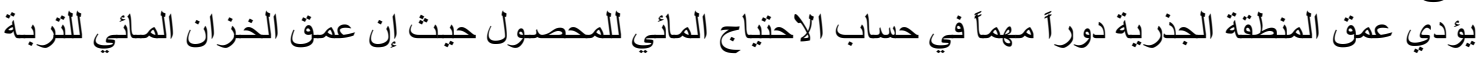

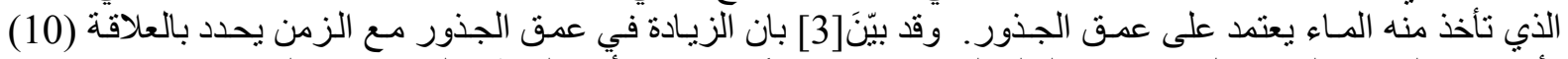

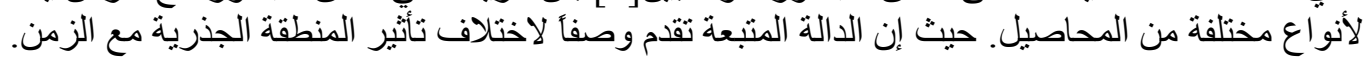

$$
Z r=Z r_{M A X}\left[0.5+0.5 \sin \left(3.03 \frac{D A P}{D T M}-1.47\right)\right]
$$

Zr

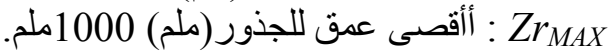
DAP DTM : عدد الأيام إلى فترة الإنضاج 61 : 61 يوم.

\section{جدولة الإرواء}

تعتمد جدولة الإرواء على توفر البيانات عن المناخ و المحصول والتربـة لمنطقة الرشيدية في الموصل. تم استخدام تربـة

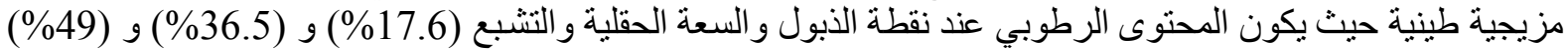

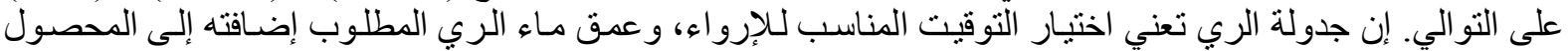

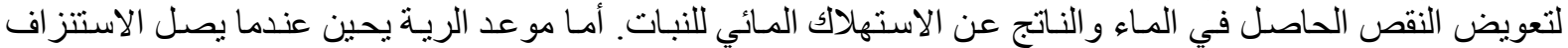

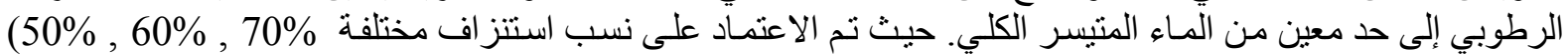

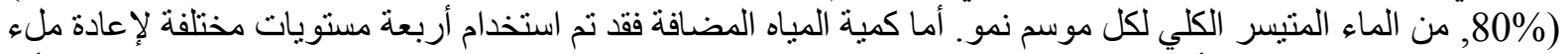

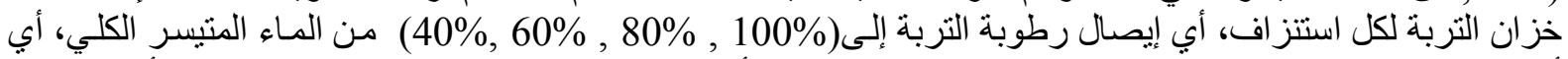

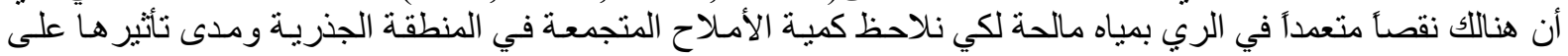

\section{النتائج والمناقشة}

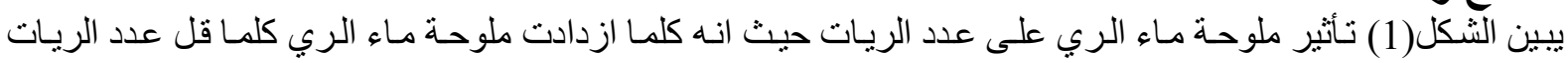

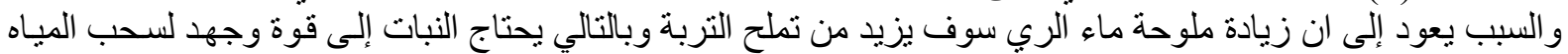

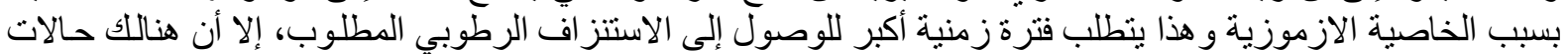

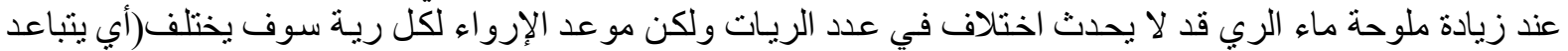

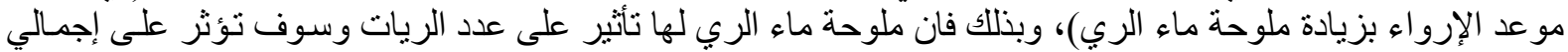

كمية المياه المضافة.

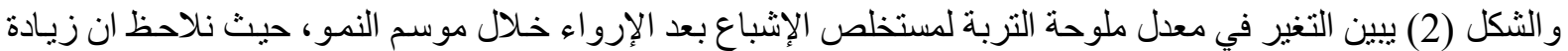

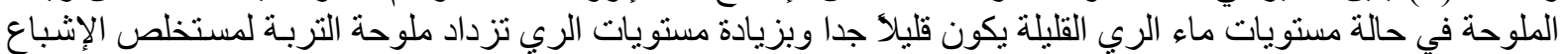

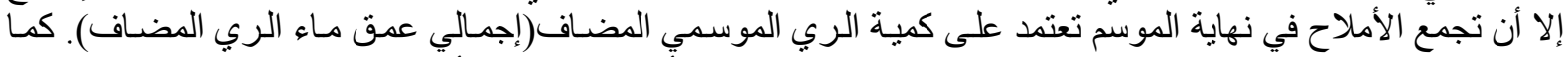

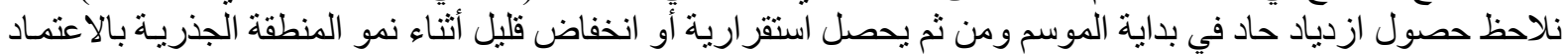

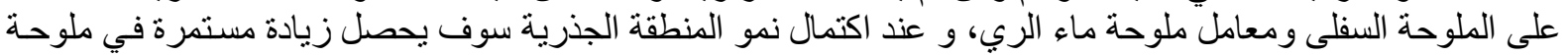

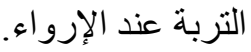




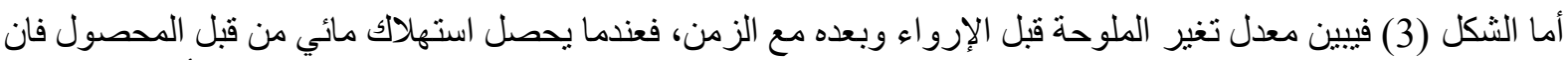

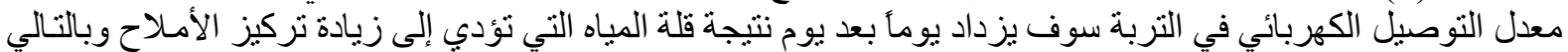

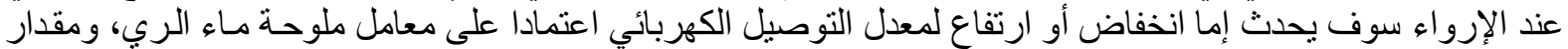

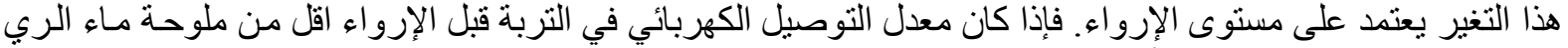

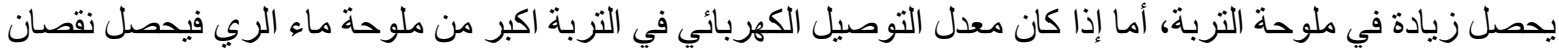

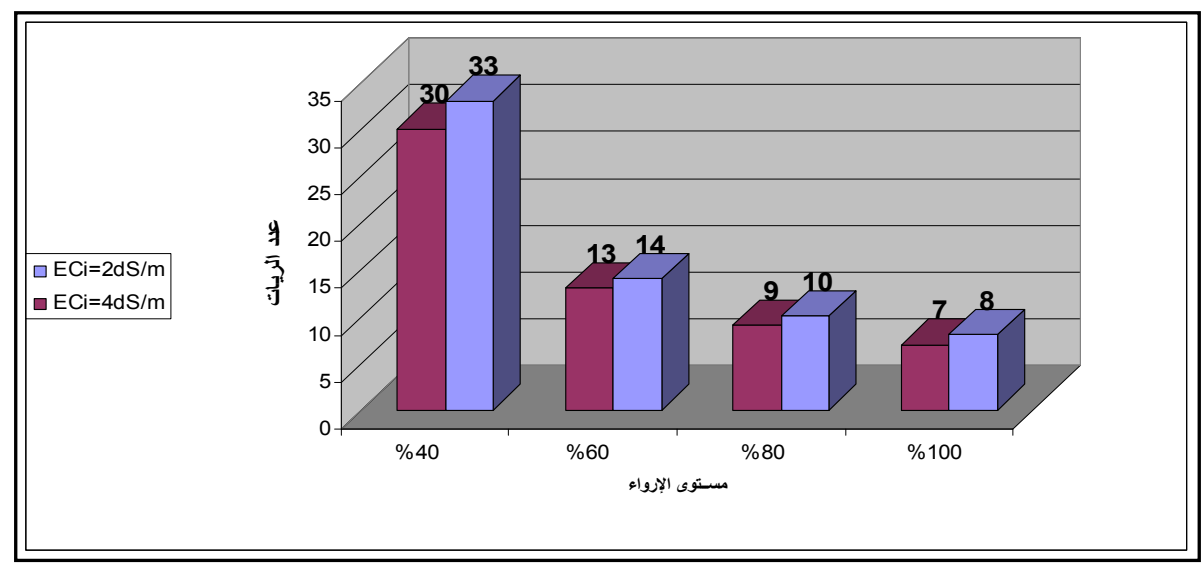

الشكل (1): تغير عدد الريات مع مستوى الإرواء عند مستويين لملوحة ماء الري

وباستنز اف رطوبي 70\% للارة.


الثكل (2): العلاقة بين معدل ملوحة التربة لمستخلص الإثباع بعد الإرواء مع

الزمن عند استنز (ف 80\% لمستويات مختلفة من الإرواء لمحصول الذرة. 

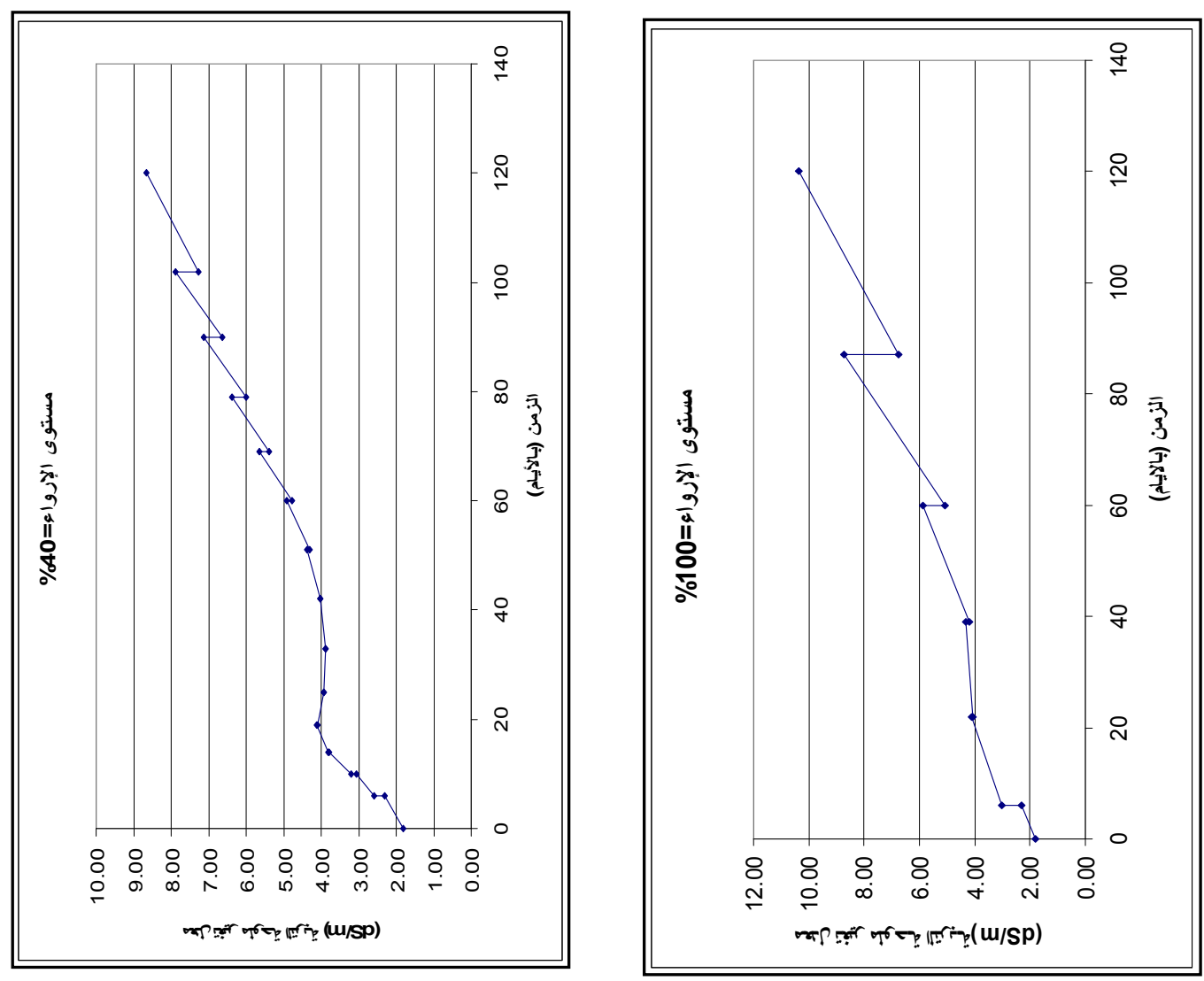

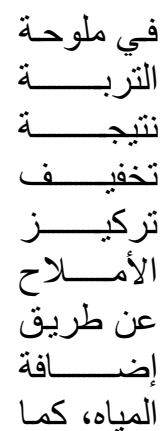
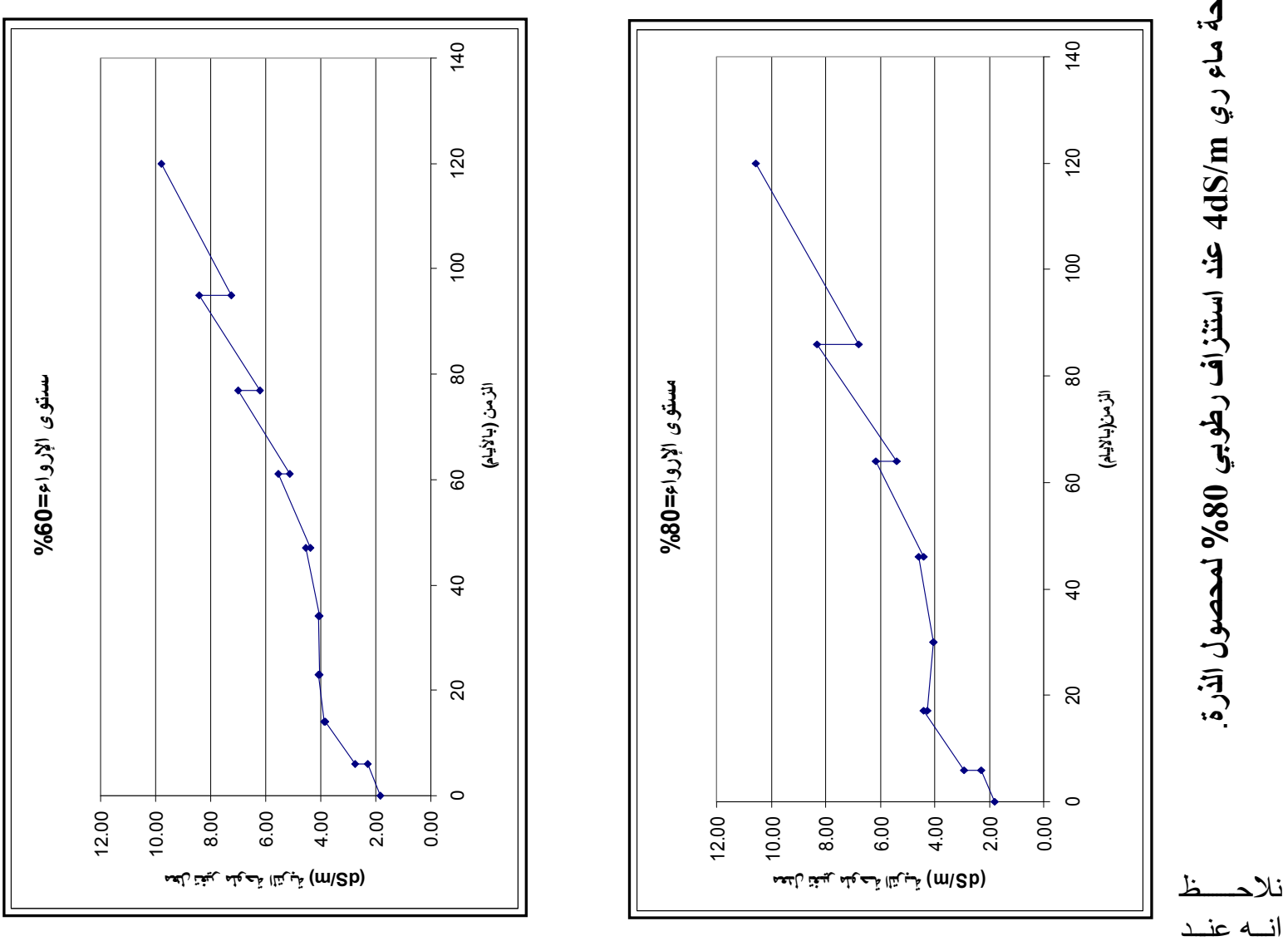
زيادة عمق المنطقة الجذرية يكون معدل تغير الملوحة قبل الإرواء وبعده قليلا نتيجة نأثرهـا بملوحة التربـة السفلى أثنـاء النمو. زادة.

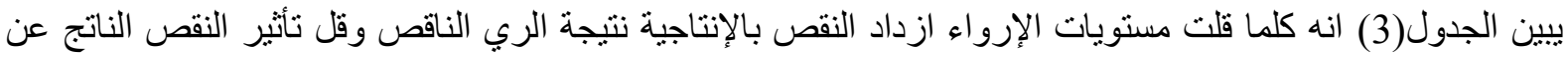

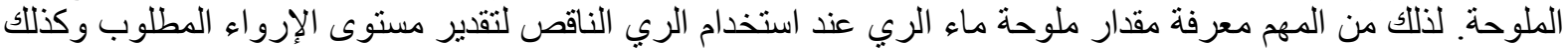

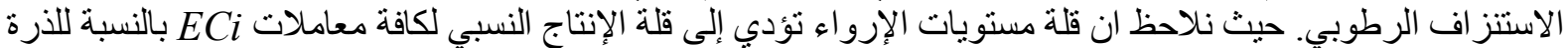

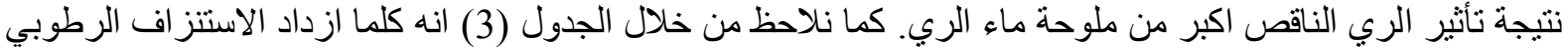

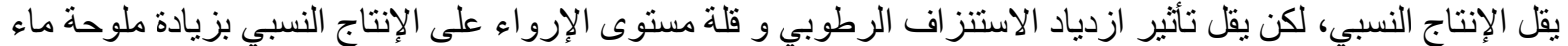

الجدول (3): نتائج الأنموذج الحاسوبي لعدة مستويات من الإرواء والاستنزاف وملوحة ماء الري لملوحة أولية $1 \mathrm{dS} / \mathrm{m}$

\begin{tabular}{|c|c|c|c|c|c|c|c|c|}
\hline الموسمبي & $\begin{array}{l}\mathbf{E T}_{\mathbf{c}} / \\
\mathbf{E T}_{\text {cadj }}\end{array}$ & لملوحة التربة المبنداع نهاية & الاستئلاك & كمية الريمي & الريات & $\begin{array}{c}\text { ملوحة ماء } \\
\text { (dS/m) }\end{array}$ & الرطنتزافي & مستوى \\
\hline 0.65 & 0.72 & 3.58 & 578 & 632 & 6 & 2 & $80 \%$ & $100 \%$ \\
\hline 0.62 & 0.69 & 3.2 & 553 & 543 & 7 & 2 & $80 \%$ & $80 \%$ \\
\hline 0.54 & 0.63 & 2.95 & 507 & 478 & 9 & 2 & $80 \%$ & $60 \%$ \\
\hline 0.41 & 0.53 & 2.5 & 422 & 368 & 14 & 2 & $80 \%$ & $40 \%$ \\
\hline 0.61 & 0.69 & 4.03 & 549 & 495 & 5 & 3 & $80 \%$ & $100 \%$ \\
\hline 0.56 & 0.65 & 4.33 & 521 & 544 & 7 & 3 & $80 \%$ & $80 \%$ \\
\hline 0.50 & 0.60 & 3.95 & 477 & 483 & 9 & 3 & $80 \%$ & $60 \%$ \\
\hline 0.39 & 0.52 & 3.23 & 412 & 365 & 14 & 3 & $80 \%$ & $40 \%$ \\
\hline 0.57 & 0.66 & 5.03 & 525 & 493 & 5 & 4 & $80 \%$ & $100 \%$ \\
\hline 0.52 & 0.62 & 4.5 & 493 & 434 & 6 & 4 & $80 \%$ & $80 \%$ \\
\hline 0.47 & 0.57 & 4.3 & 458 & 403 & 8 & 4 & $80 \%$ & $60 \%$ \\
\hline 0.37 & 0.49 & 3.7 & 393 & 335 & 13 & 4 & $80 \%$ & $40 \%$ \\
\hline 0.94 & 0.95 & 2.64 & 759.4 & 804.2 & 12 & 1 & $50 \%$ & $100 \%$ \\
\hline 0.93 & 0.94 & 2.53 & 752.6 & 753 & 18 & 1 & $50 \%$ & $80 \%$ \\
\hline 0.89 & 0.91 & 2.47 & 725.5 & 718.2 & 45 & 1 & $50 \%$ & $60 \%$ \\
\hline 0.85 & 0.88 & 3.9 & 701.12 & 711.14 & 11 & 2 & $50 \%$ & $100 \%$ \\
\hline 0.85 & 0.88 & 3.86 & 701.1 & 701 & 17 & 2 & $50 \%$ & $80 \%$ \\
\hline 0.81 & 0.85 & 3.78 & 679.06 & 681.6 & 44 & 2 & $50 \%$ & $60 \%$ \\
\hline 0.76 & 0.81 & 5.25 & 647.5 & 694.6 & 11 & 3 & $50 \%$ & $100 \%$ \\
\hline 0.76 & 0.81 & 5.25 & 648.6 & 694 & 17 & 3 & $50 \%$ & $80 \%$ \\
\hline 0.74 & 0.79 & 4.88 & 631.76 & 634 & 42 & 3 & $50 \%$ & $60 \%$ \\
\hline 0.68 & 0.74 & 6.2 & 595.1 & 637.1 & 10 & 4 & $50 \%$ & $100 \%$ \\
\hline 0.69 & 0.75 & 6.2 & $\begin{array}{l}597.8 \\
\end{array}$ & 638.7 & 16 & 4 & $50 \%$ & $80 \%$ \\
\hline 0.67 & 0.74 & 5.76 & 588.6 & 583.1 & 40 & 4 & $50 \%$ & $60 \%$ \\
\hline 0.90 & 0.92 & 2.64 & 736.2 & 801.8 & 10 & 1 & $60 \%$ & $100 \%$ \\
\hline 0.87 & 0.9 & 2.43 & 718 & 701 & 13 & 1 & $60 \%$ & $80 \%$ \\
\hline 0.81 & 0.85 & 2.38 & 677.65 & 677.3 & 24 & 1 & $60 \%$ & $60 \%$ \\
\hline 0.81 & 0.85 & 3.84 & 680 & 695.6 & 9 & 2 & $60 \%$ & $100 \%$ \\
\hline 0.80 & 0.84 & 3.85 & 672 & 700.3 & 13 & 2 & $60 \%$ & $80 \%$ \\
\hline 0.75 & 0.80 & 3.6 & 638 & 637.1 & 23 & 2 & $60 \%$ & $60 \%$ \\
\hline 0.74 & 0.79 & 5.3 & 631.4 & 696.6 & 9 & 3 & $60 \%$ & $100 \%$ \\
\hline 0.72 & 0.78 & 4.9 & 621.9 & 637 & 12 & 3 & $60 \%$ & $80 \%$ \\
\hline 0.68 & 0.75 & 4.7 & 596.6 & 598.3 & 22 & 3 & $60 \%$ & $60 \%$ \\
\hline 0.66 & 0.73 & 5.76 & 583.3 & 583.6 & 8 & 4 & $60 \%$ & $100 \%$ \\
\hline 0.66 & 0.73 & 6 & 583.3 & 619 & 12 & 4 & $60 \%$ & $80 \%$ \\
\hline 0.62 & 0.70 & 5.4 & 558.7 & 538.6 & 20 & 4 & $60 \%$ & $60 \%$ \\
\hline
\end{tabular}




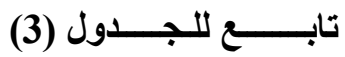

\begin{tabular}{|c|c|c|c|c|c|c|c|c|}
\hline الالدوستسي النسبي & $\begin{array}{l}\mathbf{E T}_{\mathbf{c}} / \\
\mathbf{E T}_{\text {cadj }}\end{array}$ & الملوحة التربة الإشباع نهاية & المائي الحقيقي & الموسمية الرئلم) & الريات & $\begin{array}{l}\text { ملوحة الري } \\
\text { (dS/m) }\end{array}$ & الرطنتزبي & مستوى \\
\hline 0.82 & 0.86 & 2.5 & 686.5 & 735.6 & 8 & 1 & $70 \%$ & $100 \%$ \\
\hline 0.79 & 0.83 & 2.3 & 663.6 & 658 & 10 & 1 & $70 \%$ & $80 \%$ \\
\hline 0.70 & 0.76 & 2.18 & 609 & 575.8 & 14 & 1 & $70 \%$ & $60 \%$ \\
\hline 0.56 & 0.65 & 1.99 & 518.3 & 487 & 34 & 1 & $70 \%$ & $40 \%$ \\
\hline 0.76 & 0.81 & 4 & 647.8 & 732.1 & 8 & 2 & $70 \%$ & $100 \%$ \\
\hline 0.73 & 0.78 & 3.7 & 626.4 & 657 & 10 & 2 & $70 \%$ & $80 \%$ \\
\hline 0.66 & 0.73 & 3.3 & 581.3 & 570 & 14 & 2 & $70 \%$ & $60 \%$ \\
\hline 0.53 & 0.63 & 2.9 & 500 & 469 & 33 & 2 & $70 \%$ & $40 \%$ \\
\hline 0.69 & 0.75 & 4.7 & 598.4 & 605.7 & 7 & 3 & $70 \%$ & $100 \%$ \\
\hline 0.66 & 0.73 & 4.5 & 583.6 & 573 & 9 & 3 & $70 \%$ & $80 \%$ \\
\hline 0.61 & 0.69 & 4.1 & 550 & 506.8 & 13 & 3 & $70 \%$ & $60 \%$ \\
\hline 0.50 & 0.60 & 3.73 & 478.7 & 446.4 & 32 & 3 & $70 \%$ & $40 \%$ \\
\hline 0.62 & 0.70 & 6 & 555.85 & 611.3 & 7 & 4 & $70 \%$ & $100 \%$ \\
\hline 0.61 & 0.69 & 5.7 & 547.6 & 580 & 9 & 4 & $70 \%$ & $80 \%$ \\
\hline 0.56 & 0.65 & 5.2 & 517.4 & 512.6 & 13 & 4 & $70 \%$ & $60 \%$ \\
\hline 0.46 & 0.57 & 4.4 & 455.9 & 415.8 & 30 & 4 & $70 \%$ & $40 \%$ \\
\hline 0.70 & 0.76 & 2.3 & 609 & 635 & 6 & 1 & $80 \%$ & $100 \%$ \\
\hline 0.67 & 0.73 & 2.1 & 587 & 539 & 7 & 1 & $80 \%$ & $80 \%$ \\
\hline 0.57 & 0.66 & 1.97 & 526 & 476 & 9 & 1 & $80 \%$ & $60 \%$ \\
\hline 0.42 & 0.54 & 1.75 & 428 & 368 & 14 & 1 & $80 \%$ & $40 \%$ \\
\hline
\end{tabular}

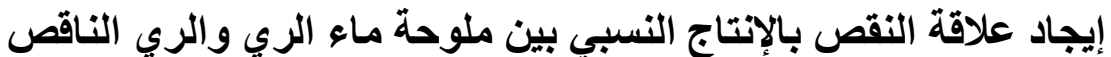

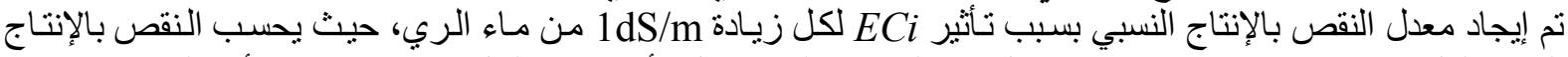

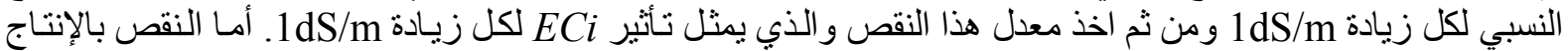

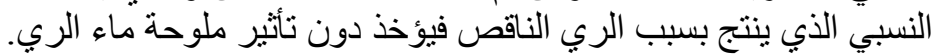

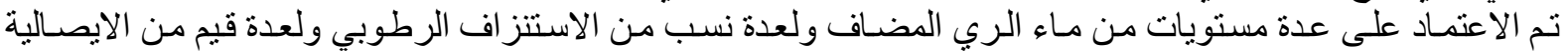

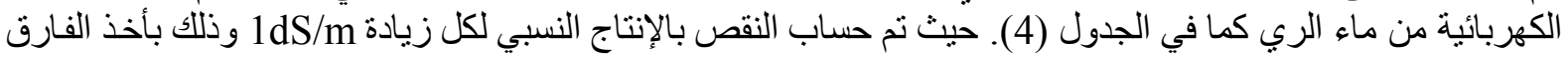

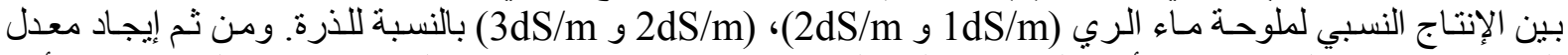

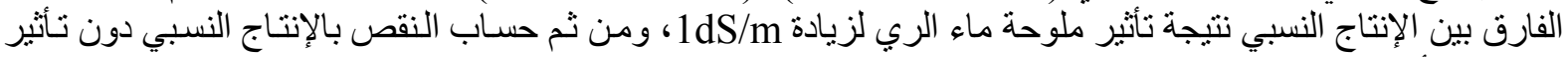

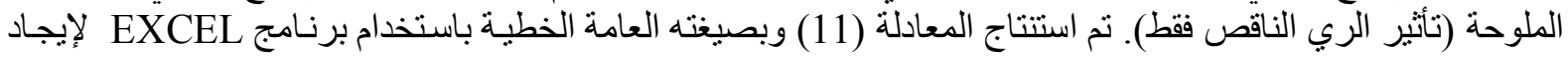

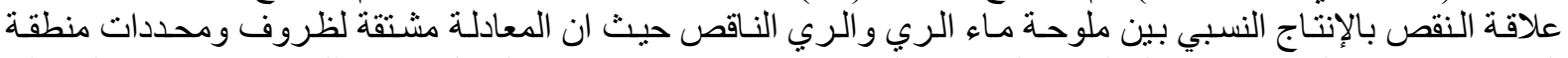

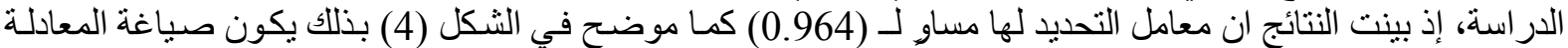
بشكل عام كالآتي:

$Y=\left(E C i-E C i_{\text {active }}\right)(A X+B)$

ويمكن حساب مقدار الإنتاج النسبي بسبب ECi والري الناقص من خلال المعادلة (12) إذا علم مقدار نسبة النقص بالإنتاج نتيجة الري الناقص من دون تأثير ECi

$\frac{Y_{a}}{Y_{m}}=1-(Y+X)$

وبتعويض المعادلة (11) بـ(12) ينتج (13)

$\frac{Y_{a}}{Y_{m}}=1-\left(\left(E C i-E C i_{\text {cative }}\right) \times(A X+B)+X\right)$ 
حيث ان:

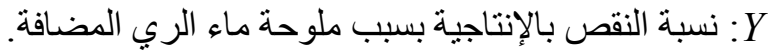

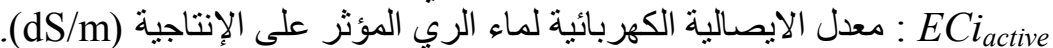
: : $B$ ، : : $A$ X

\section{الجدول (4): علاقة النقص بالإنتاج النسبي بين ECi و الري الناقص لعدة مستويات من ماء الري و ECi مختلف}

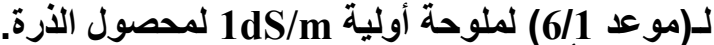

كما يمكن حساب التبخر -نتح الحقيقي نتيجة تأثير ملوحة ماء الري وقلة الرئة المباه إذا علم التبخر - نتح الحقيقي الموسمي نتيجة

\begin{tabular}{|c|c|c|c|c|c|c|c|c|c|}
\hline النالنتب النقب & 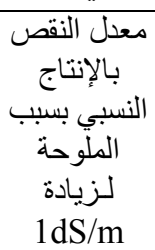 & $\begin{array}{c}\text { النسالإتناج لـ الفرق } \\
\text { النسبي } \\
\text { ECi=3 } \\
\text { ECi=2 } \\
\text { dS/m }\end{array}$ & 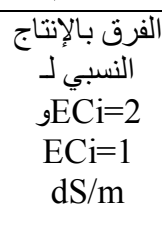 & $\begin{array}{l}\text { النسبئل الإنتاج } \\
\text { ECi=3 } \\
\text { dS/m }\end{array}$ & 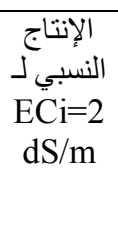 & 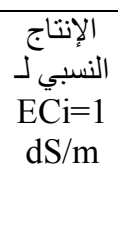 & \begin{tabular}{|c|c|} 
الإنتاج النسبي \\
$\mathrm{ECi}=0.2 \mathrm{dS} / \mathrm{m}$
\end{tabular} & الاستنز اف & الإرواء \\
\hline 0.03 & 0.09 & 0.08 & 0.09 & 0.76 & 0.85 & 0.94 & 0.97 & 0.5 & 1 \\
\hline 0.03 & 0.09 & 0.09 & 0.09 & 0.76 & 0.85 & 0.94 & 0.97 & 0.5 & 0.9 \\
\hline 0.04 & 0.08 & 0.08 & 0.08 & 0.76 & 0.85 & 0.93 & 0.96 & 0.5 & 0.8 \\
\hline 0.05 & 0.08 & 0.08 & 0.08 & 0.76 & 0.84 & 0.92 & 0.95 & 0.5 & 0.7 \\
\hline 0.09 & 0.07 & 0.07 & 0.07 & 0.74 & 0.81 & 0.89 & 0.91 & 0.5 & 0.6 \\
\hline 0.07 & 0.08 & 0.08 & 0.09 & 0.74 & 0.81 & 0.90 & 0.93 & 0.6 & 1 \\
\hline 0.08 & 0.08 & 0.08 & 0.07 & 0.73 & 0.81 & 0.89 & 0.92 & 0.6 & 0.9 \\
\hline 0.10 & 0.07 & 0.08 & 0.07 & 0.72 & 0.80 & 0.87 & 0.90 & 0.6 & 0.8 \\
\hline 0.13 & 0.07 & 0.07 & 0.06 & 0.71 & 0.78 & 0.84 & 0.87 & 0.6 & 0.7 \\
\hline 0.17 & 0.06 & 0.06 & 0.06 & 0.68 & 0.75 & 0.81 & 0.83 & 0.6 & 0.6 \\
\hline 0.24 & 0.05 & 0.06 & 0.05 & 0.64 & 0.69 & 0.75 & 0.76 & 0.6 & 0.5 \\
\hline 0.15 & 0.07 & 0.07 & 0.06 & 0.69 & 0.76 & 0.82 & 0.85 & 0.7 & 1 \\
\hline 0.17 & 0.07 & 0.07 & 0.07 & 0.67 & 0.74 & 0.81 & 0.83 & 0.7 & 0.9 \\
\hline 0.20 & 0.06 & 0.07 & 0.06 & 0.66 & 0.73 & 0.79 & 0.80 & 0.7 & 0.8 \\
\hline 0.24 & 0.05 & 0.05 & 0.05 & 0.64 & 0.70 & 0.75 & 0.76 & 0.7 & 0.7 \\
\hline 0.29 & 0.05 & 0.05 & 0.04 & 0.61 & 0.66 & 0.70 & 0.71 & 0.7 & 0.6 \\
\hline 0.36 & 0.04 & 0.04 & 0.04 & 0.56 & 0.60 & 0.64 & 0.64 & 0.7 & 0.5 \\
\hline 0.44 & 0.03 & 0.03 & 0.03 & 0.50 & 0.53 & 0.56 & 0.56 & 0.7 & 0.4 \\
\hline 0.28 & 0.05 & 0.04 & 0.05 & 0.61 & 0.65 & 0.70 & 0.72 & 0.8 & 1 \\
\hline 0.30 & 0.04 & 0.06 & 0.03 & 0.60 & 0.65 & 0.68 & 0.70 & 0.8 & 0.9 \\
\hline 0.33 & 0.05 & 0.05 & 0.05 & 0.56 & 0.62 & 0.67 & 0.67 & 0.8 & 0.8 \\
\hline 0.37 & 0.04 & 0.05 & 0.04 & 0.54 & 0.59 & 0.62 & 0.63 & 0.8 & 0.7 \\
\hline 0.42 & 0.04 & 0.05 & 0.03 & 0.50 & 0.54 & 0.57 & 0.58 & 0.8 & 0.6 \\
\hline 0.49 & 0.03 & 0.04 & 0.02 & 0.44 & 0.49 & 0.51 & 0.51 & 0.8 & 0.5 \\
\hline 0.58 & 0.01 & 0.02 & 0.01 & 0.39 & 0.41 & 0.42 & 0.42 & 0.8 & 0.4 \\
\hline
\end{tabular}

قلة المياه و التبخر - نتح للمحصول الموسمي من خلال المعادلة (14) و الناتجة من تعويض معادلة النقص بالإنتاج النسبي (9) بمعادلة (13) (لبخر (13)

$$
K y\left(1-\frac{E T_{\text {cadj }}}{E T_{c}}\right)=\left(\left(E C i-E C i_{\text {active }}\right) \times\left(A \times K y\left(1-\frac{E T_{\text {cadj } B}}{E T_{c}}\right)+B\right)+K y\left(1-\frac{E T_{\text {cadj } B}}{E T_{c}}\right)\right)
$$

ET cadjA ET cadjB 


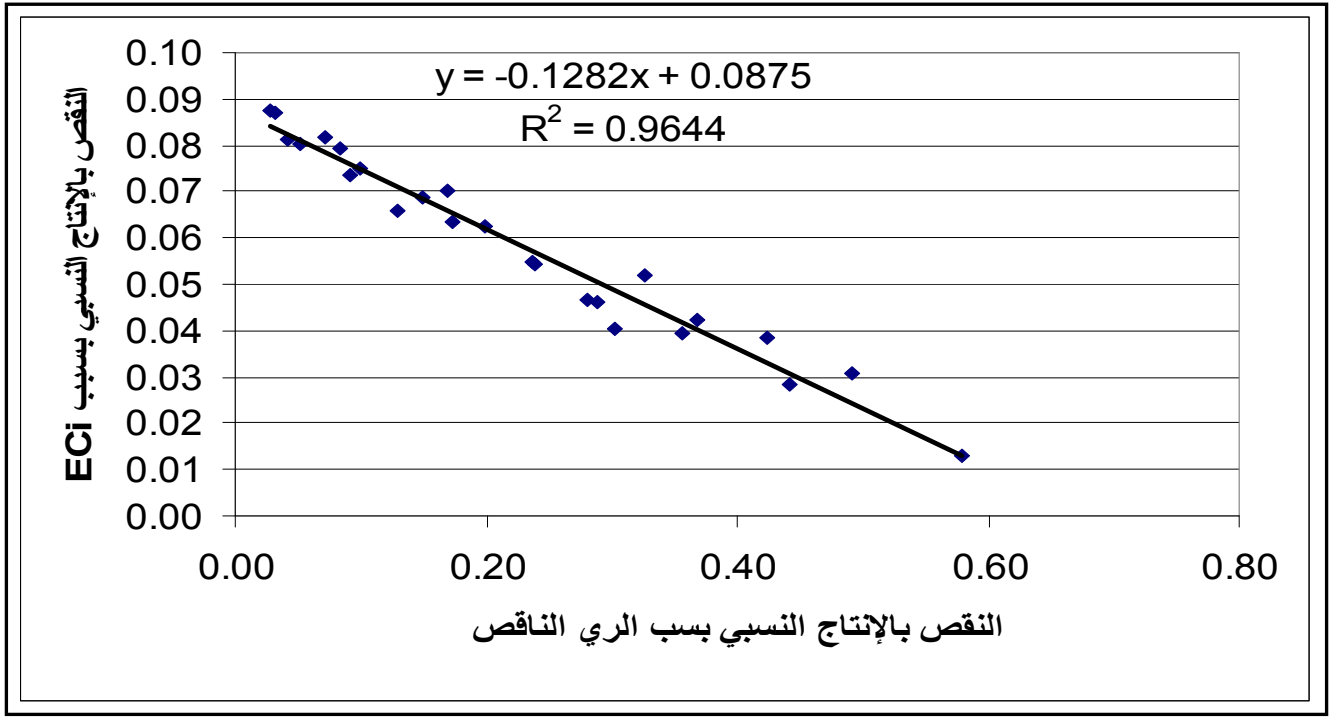

الثكل (4): علاقة النقص بالإنتاج النسبي بين ملوحة ماء الري والري الناقص لمحصول الذرة.

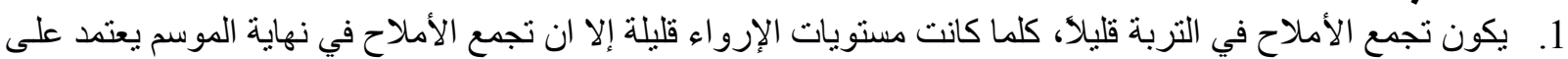

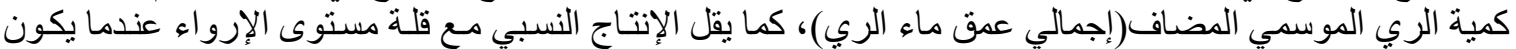

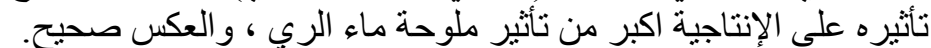

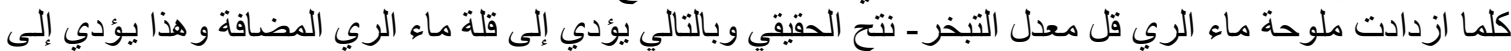

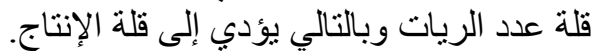

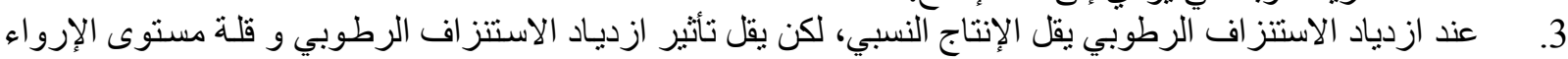

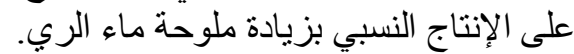

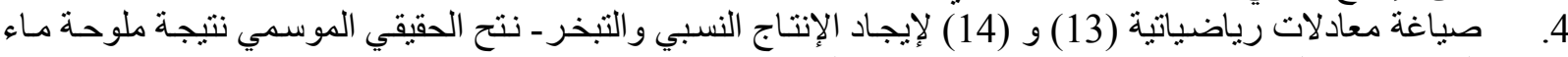
الري ونقص المياه تحت ظروف ومحددات منطقة الدراسة.

1. محمود، عمر مقداد عبد الغني، (2009). " أنموذج حاسوبي لمحاكاة تأثير الري الناقص بمياه مالحة على ملوحة التربة و الإنتاجية "، رسالة ماجستير ، جامعة الموصل، كلية الهندسة.

2. Allen, R. G., Pereira, L. S., Raes, D. and Smith, M., (1998). " Crop Evapotranspiration-Guidelines for Computing Crop Water Requirements ". FAO Irrigation and Drainage paper No.56, Rome, Italy

3. Borg, H. and Grimes, D.W., (1986)."Depth Development of Roots with Time ".Trans. ASAE. 29(1): 194-197.

4. Doorenbos, J. and Kassam, A. H., (1979). "Yield Response to Water". FAO Irrigation and Drainage Paper No. 33. 
5. Henggeler, J. C., (2004). " The Conjunctive use of Saline Irrigation Water on Deficit-Irrigation Cotton ". Doctor of Philosophy thesis, the Office of Graduate Studies of Texas A\&M University.

6. Ozturk, A., Unlukara, A., Ipek, A. and Gurbuz, B., (2004). "Effects of Salt Stress and Water Deficit on Plant Growth and Essential Oil Content of Lemon Balm". Pak. J. Bot. 36(4): 787-792

7. Sezen, M. S., Yazar, A., Akyildiz, A., Dasgan H. Y. and Gencel, B., (2008). "Yield and Quality Response of Drip Irrigated Green Beans Under Full and Deficit Irrigation". WWW. elsevier. Com/locate/sichort., Scientia Horticulturae. 117 : 95102.

8. Tilman, D. K., Cassman, G., Matson, P. A., Naylor, R. and Polasky, S. (2002). " Review Article Agricultural Sustainability and Intensive Production Practices ". Nature 418: 671-

$$
\text { تم اجراء البحث في كلية ألهندسة = جامعة ألموصل }
$$

